\title{
La révolution \\ des exoplanètes
}

\author{
J. Lequeux \\ T. Encrenaz \\ F. Casoli
}

\section{edpsciences}

17, avenue du Hoggar

Parc d'activités de Courtaboeuf, BP 112

91944 Les Ulis Cedex A, France 



\section{Dans la même collection}

Les planètes : les nôtres et les autres

Thérèse Encrenaz

Naissance, évolution et mort des étoiles

James Lequeux

La fusion thermonucléaire contrôlée

Jean-Louis Bobin

Le nucléaire expliqué par des physiciens

Bernard Bonin, préface d'É. Klein

Mathématiques des marchés financiers

Mathieu Le Bellac et Arnaud Viricel, préface de J.-Ph. Bouchaud

Physique et biologie

Jean-François Allemand et Pierre Desbiolles

La cryptologie

Philippe Guillot

L'aventure du grand collisionneur LHC

Daniel Denegri, Claude Guyot, Andreas Hoecker et Lydia Roos, préface de C. Rubbia

Le climat : la Terre et les hommes

Jean Poitou, Pascale Braconnot et Valérie Masson-Delmotte, préface de J. Jouzel

Aux origines de la masse : particules élémentaires et symétrie fondamentales

Jean Iliopoulos, préface de F. Englert

Les relativités : espace, temps, gravitation

Michel Le Bellac

Retrouvez tous nos ouvrages et nos collections sur http:/ /laboutique.edpsciences.fr

\section{Imprimé en France.}

(C) 2017, EDP Sciences, 17, avenue du Hoggar, BP 112, Parc d'activités de Courtaboeuf, 91944 Les Ulis Cedex A

Tous droits de traduction, d'adaptation et de reproduction par tous procédés réservés pour tous pays. Toute reproduction ou représentation intégrale ou partielle, par quelque procédé que ce soit, des pages publiées dans le présent ouvrage, faite sans l'autorisation de l'éditeur est illicite et constitue une contrefaçon. Seules sont autorisées, d'une part, les reproductions strictement réservées à l'usage privé du copiste et non destinées à une utilisation collective, et d'autre part, les courtes citations justifiées par le caractère scientifique ou d'information de l'œuvre dans laquelle elles sont incorporées (art. L. 122-4, L. 122-5 et L. 335-2 du Code de la propriété intellectuelle). Des photocopies payantes peuvent être réalisées avec l'accord de l'éditeur. S'adresser au : Centre français d'exploitation du droit de copie, 3, rue Hautefeuille, 75006 Paris. Tél. : 0143269535.

ISBN (papier) 978-2-7598-2111-2 - ISBN (ebook) 978-2-7598-2148-8 
